\title{
Correction to: Prevalence of HIV-1 transmitted drug resistance in recently infected, treatment-naïve persons in the Southwest of Iran, 2014-2015
}

\author{
Shokouh Ghafari ${ }^{1,2} \cdot$ Arash Memarnejadian $^{3}$ - Alireza Samarbaf-zadeh ${ }^{1,2}$. Ehsan Mostafavi ${ }^{4,5}$. \\ Manoochehr Makvandi ${ }^{1,2}$. Shokrolah Salmanzadeh ${ }^{2}$. Ata Ghadiri ${ }^{6}$ Michael R. Jordan ${ }^{7}$. Elham Mousavi ${ }^{1,2}$. \\ Fatemeh Jahanbakhsh ${ }^{8} \cdot$ Kayhan Azadmanesh $^{9}$
}

Published online: 4 December 2017

(c) Springer-Verlag GmbH Austria, part of Springer Nature 2017

\section{Correction to: Arch Virol (2017) 162:2737-2745 https://doi.org/10.1007/s00705-017-3431-0}

The author would like to correct the error in the online published article. The correct details are given below for your reading:

Eleventh sentence of the section "Study design and sample collection" should read as:

"HIV-1 infection of the participants was diagnosed through the application of a commercial HIV-1 rapid test strip followed by both ELISA and Western blot confirmatory tests, in accordance with the HIV national algorithms in Iran."

The original article can be found online at https://doi. org/10.1007/s00705-017-3431-0.

Alireza Samarbaf-zadeh

alirezasamarbaf_78@hotmail.com

1 Health Research Institute, Infectious and Tropical Disease Research Center, Ahvaz Jundishapur University of Medical Sciences, Ahvaz, Iran

2 Department of Virology, School of Medicine, Ahvaz Jundishapur University of Medical Sciences, Ahvaz, Iran

3 Hepatitis and AIDS Department, Pasteur Institute of Iran, Tehran, Iran

4 Department of Epidemiology, Pasteur Institute of Iran, Tehran, Iran
5 Research Centre for Emerging and Reemerging Infectious Diseases, Pasteur Institute of Iran, Akanlu, Kabudar-Ahang, Hamadan, Iran

6 Cellular and Molecular Research Center, Faculty of Medicine, Ahvaz Jundishapur University of Medical Sciences, Ahvaz, Iran

7 Tufts University School of Medicine, Boston, USA

8 Virology Research Group, Pasteur institute of Iran, Tehran, Iran

9 Department of Virology, Pasteur Institute of Iran, Tehran, Iran 